\title{
Avaliação do bem-estar de cuidadores familiares de adultos com neoplasia maligna no âmbito domiciliar
}

\section{Evaluation of the well-being of family caregivers of adults with malignant neoplasm in the home environment}

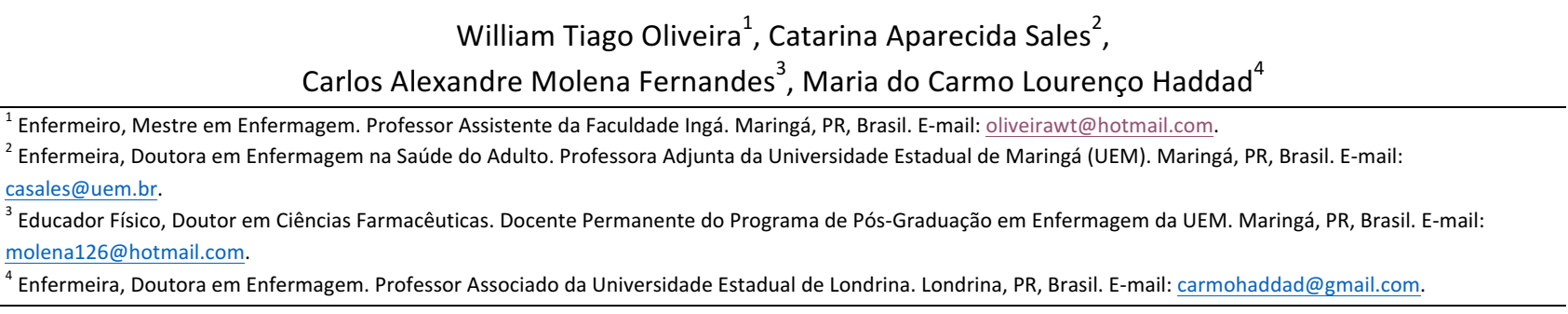

\section{RESUMO}

O objetivo deste estudo foi analisar o bem-estar de cuidadores familiares de adultos com neoplasias malignas no âmbito domiciliar pelo Questionário de Avaliação do Bem-Estar Global. Trata-se de um estudo transversal, desenvolvido no município de Maringá-PR, com 96 cuidadores. A coleta de dados foi realizada entre julho de 2011 e fevereiro de 2012. Os resultados foram analisados por meio de estatística descritiva. A presença da espiritualidade e os benefícios quando se tem a presença da rede de apoio oferecendo suporte na prestação dos cuidados, são fatores que mais corroboraram para promoção do bem-estar. As preocupações com a família e o desconforto físico e emocional do doente, foram os itens que tiveram os menores escores. Ao comparar os resultados com estudo realizado em âmbito hospitalar, concluiuse que o cenário interfere pouco em relação ao bem-estar. No entanto, alguns itens sugerem que cuidar no domicílio tem impacto maior no bem-estar dos cuidadores.

Descritores: Enfermagem; Neoplasias; Família; Cuidadores; Qualidade de Vida.

\section{ABSTRACT}

The aim of this study was to analyze the well-being of family caregivers of adults with malignant neoplasms in the home environment by means of the Avaliação de Bem-Estar Global (a validated version of the General Comfort Questionnaire for Brazilian Portuguese). A cross-sectional study was developed in Maringá, in the state of Paraná, comprising 96 caregivers. Data were collected between July 2011 and February 2012. Results were analyzed using descriptive statistics. The presence of spirituality and the benefits of a network offering support for care provision are factors that contributed to promoting well-being. Family concerns and physical and emotional discomfort of the patient were the items that had the lowest scores. When comparing the results with a study developed in a hospital environment, we concluded that the scenario has little effect on well-being. However, some items suggest care provided at home has a greater impact on the well-being of caregivers.

Descriptors: Nursing; Neoplasms; Family; Caregivers; Quality of Life. 


\section{INTRODUÇÃO}

As repercussões causadas pelo câncer ao paciente e a sua família são bem conhecidas, havendo, na atualidade, uma tendência para que seja transferida para a família a responsabilidade com os cuidados do paciente ${ }^{(1)}$. Pelo processo natural, a pessoa com câncer torna-se gradativamente mais dependente de cuidados. Essa necessidade culmina no surgimento de um cuidador, que é a pessoa responsável por prover os cuidados ao doente ${ }^{(2)}$.

A literatura tem abordado a respeito da sobrecarga do cuidador e sua estafante e estressora atividade de cuidados. Há, nesta área de estudos, um número significativo de trabalhos que se relacionam à deterioração de sua saúde e adoecimento, tanto físico quanto psíquico ${ }^{(3-4)}$. Face à gravidade da situação, o cuidador necessita de intervenções que promovam seu bem-estar, proporcionando adaptação às condições geradas pela doença ${ }^{(5)}$.

Um caminho alternativo para estudar e identificar os principais problemas ou necessidades dos cuidadores é a utilização de instrumentos já testados. Há muitos instrumentos padronizados disponíveis na literatura nesta linha, porém a maioria não é completa em relação a todas as dimensões dos cuidados (físico, psicológico, social, econômicas e espiritual) ${ }^{(6)}$.

Em um estudo de revisão crítica foram analisados alguns dos principais instrumentos padronizados $e$ disponíveis na literatura utilizados para avaliar a qualidade de vida do cuidador de pacientes com câncer e apontou o General Comfort Questionnaire (GCQ), criado a partir da teoria do conforto, desenvolvida pela enfermeira americana Katherine Kolcaba ${ }^{(7)}$, como uma ferramenta útil à prática clínica, pois possibilita a avaliação multidimensional (físico, ambiental, social, psicológico e espiritual) e permite identificar os diversos fatores que diminuem ou elevam o bem-estar do cuidador $^{(6)}$.

O referido questionário foi utilizada, no Brasil, pela primeira vez, junto a 133 cuidadores de pacientes internados na enfermaria oncológica de um hospital público $^{(8)}$, e sua versão para o português recebeu o título de Avaliação do Bem-Estar Global (BEG). Outros estudos também se utilizaram deste instrumento, mas nenhum foi aplicado há cuidadores de pacientes com câncer no âmbito domiciliar ${ }^{(9-10)}$.

Assim, justifica-se a realização deste estudo que teve como objetivo avaliar o bem-estar global de cuidadores familiares de adultos com neoplasia maligna no âmbito domiciliar, oportunizando que medidas preventivas e ajustes pontuais possam ser introduzidos, para minimizar o sofrimento decorrente da tarefa de ser cuidador. Vale ressaltar que a análise e discussão desse estudo foram realizadas, principalmente, por meio de comparação com os resultados encontrados em estudo realizado com o cuidador no âmbito hospitalar ${ }^{(8)}$.

\section{MATERIAL E MÉTODO}

Estudo descritivo, transversal, desenvolvido no município de Maringá-PR, junto a familiares que cuidam de pessoas adultas com neoplasias malignas. $\mathrm{O}$ contato com esses cuidadores deu-se por meio da Rede Feminina de Combate ao Câncer (RFCC), que atende pacientes oncológicos, usuário do Sistema Único de Saúde (SUS), residentes nas cidades pertencentes à 15a Regional de Saúde do Estado do Paraná.

Os critérios de inclusão para seleção dos cuidadores foram: ter algum vínculo familiar (laços consanguíneos ou vínculos emocionais) com a pessoa com câncer; ser identificado como cuidador principal pelo próprio paciente ou com a ajuda de um familiar nos casos em que este se encontrava em condições de saúde que o impedia de fornecer a informação; ter idade maior ou igual a 18 anos; não receber remuneração salarial para cuidar; conviver diariamente com o indivíduo com câncer; prestar cuidados ao familiar com câncer há mais de dois meses; cuidador e familiar devem residir em Maringá (optou-se por excluir os casos de pessoas com câncer, cadastrados na RFCC que residiam em outras cidades, devido a dificuldade de transporte para a realização das 
entrevistas). Foram excluídos os cuidadores de crianças com câncer.

No período da coleta de dados, a RFCC continha o cadastro de 273 pacientes com neoplasias malignas. Destes, 170 residiam no município de Maringá e os demais eram de outras cidades pertencentes à 15a regional de saúde do Paraná. Dos 170 possíveis contatos, 74 foram excluídos devido a: impossibilidade de contato telefônico - número incorreto ou desativado (47); óbito (nove); mudança para outras cidades (cinco); não identificação do cuidador principal (três); cuidadores de crianças com câncer (dois); recusa em participar da pesquisa (oito). Portanto, a população final deste estudo constituiu-se de 96 cuidadores.

Para a caracterização dos participantes da pesquisa, foi elaborado um questionário, contendo 12 questões objetivas referentes às características pessoais dos familiares cuidadores.

Para avaliar o bem-estar dos cuidadores, foi utilizado o Questionário de Avaliação do Bem-Estar Global (BEG), que consiste em um instrumento de autopreenchimento, mas que pode também ser aplicado pelo pesquisador. Contém 49 perguntas que são respondidas por meio de uma escala tipo Likert, com variação numérica de um a seis, sendo que um se refere a discordo fortemente e seis concordo fortemente. Os números entre os dois extremos devem ser assinalados de acordo com o quão forte é a sensação/impressão sobre a disponibilidade ou não do recurso/condição. Vinte e seis itens são redigidos de forma negativa para evitar respostas automáticas pelo participante da pesquisa. Os itens redigidos na forma negativa foram invertidos durante a fase de tratamento dos dados, ou seja, quando apresentava a pontuação seis era convertida para um, e assim por diante ${ }^{(6)}$. O escore final do BEG é construído como uma escala começando com 49 pontos, péssimo bem-estar, e finalizando com 294 pontos, excelente bem-estar.

O referido instrumento foi testado, primeiramente, nos Estados Unidos e, recentemente, traduzido para o Brasil $^{(8)}$. A análise da versão em português atingiu Alpha de Cronbach de 0,83, o que indica a adequação da escala e uma excelente consistência interna entre seus itens.

A coleta de dados foi realizada entre os meses de julho de 2011 e fevereiro de 2012. Inicialmente, foi realizado contato, via telefone, com o paciente e/ou familiar a fim de identificar o cuidador principal. Após sua identificação, foi realizado contato com este cuidador e lançado o convite para participar da pesquisa. Em caso de aceite, foi agendada uma visita no domicílio do cuidador para a aplicação do questionário. As entrevistas foram realizadas de forma individual, em que para cada respondente, as questões do instrumento foram lidas e as respostas anotadas pelo entrevistador, a fim de contornar problemas de analfabetismo e baixa escolaridade.

Os dados obtidos por meio dos questionários foram digitados em um banco de dados no pacote estatístico Statistical Package for Social Sciences 18.0 e analisados descritivamente, com cálculo dos resultados em valores mínimos, médios e máximos, e desvio-padrão. Optou-se por apresentar os resultados desta forma, para facilitar a comparação com estudo já realizado que utilizou o BEG.

A realização deste estudo foi precedida pelo encaminhamento do projeto de pesquisa ao Comitê Permanente de Ética em Pesquisa Envolvendo Seres Humanos da Universidade Estadual de Maringá, tendo obtido parecer favorável com o nำ423/2010 e mediante registro no Sistema Nacional de Informação sobre Ética em Pesquisa com CAAE no 0171.0.093.000-10, conforme Resolução 196/1996 do Conselho Nacional da Saúde.

\section{RESULTADOS}

\section{Caracterização dos cuidadores}

Da totalidade dos 96 cuidadores que participaram do estudo, 77 eram do sexo feminino, o que correspondeu a pouco mais de $80 \%$. Em relação à distribuição das idades, verificou-se que a idade média foi de 54,9 anos $(d p=15,80)$, sendo a idade mínima de 18 anos e a máxima de 85 anos. 


\section{Avaliação do bem-estar global}

Os escores totais de bem-estar situaram entre 168 e 255 , o que indica variabilidade individual (o menor escore é $65 \%$ menor que o maior) nos níveis de bem-estar global.

Por outro lado, pode-se notar, a partir da Figura 1, que apresenta a dispersão dos escores dos participantes, em ordem crescente, no intervalo possível de pontuação na escala (49 a 294), que a maior parte dos cuidadores (71 participantes) teve seu nível de bem-estar global inserido no intervalo entre 196 e 245, ou seja, no quarto quintil da distribuição. Vale destacar que nenhum participante apresentou escore no primeiro e segundo quintil e apenas quatro participantes teve escore no quinto quintil da distribuição.

\section{Somatória dos pontos do BEG}

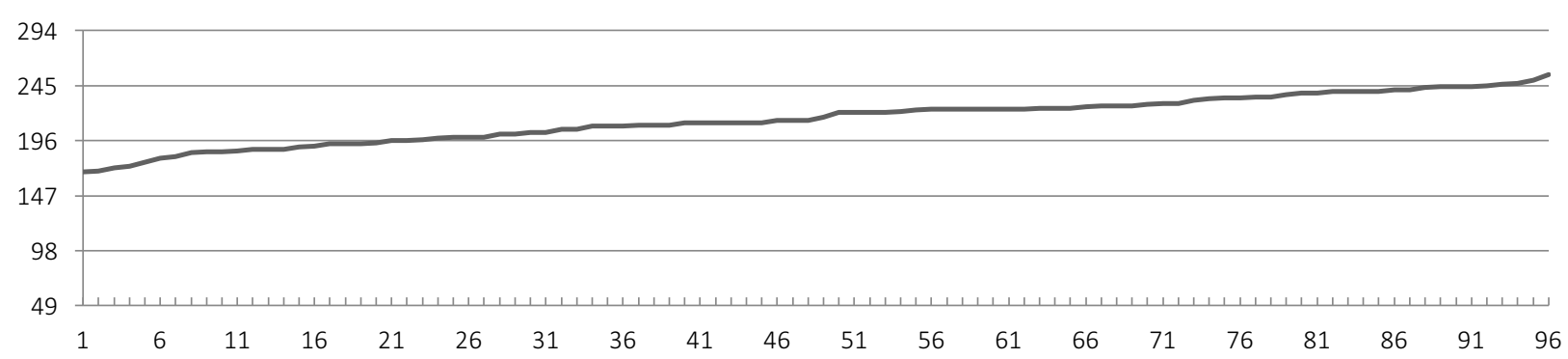

Figura 1: Dispersão dos escores do BEG. Maringá, PR, Brasil, 2012.

Os dados obtidos a partir da aplicação do Questionário de Avaliação do BEG foram organizados de modo a destacar os maiores e menores valores médios das questões da escala, sugerindo quais fatores estão mais e menos relacionados ao bem-estar dos cuidadores.

O valor médio das questões variou de 1,00 a 5,96. Na Tabela 1 são apresentados, em ordem decrescente, os valores médios mais altos das questões do BEG (situados entre 6,0 e 5,0), bem como o desvio-padrão (DP) e as variações individuais de cada resposta, a partir dos valores mínimos e máximos atribuídos a cada questão.

Os fatores que mais contribuíram para diminuir o bem-estar dos cuidadores podem ser observados na Tabela 2, que apresenta, em ordem crescente, os valores médios mais baixos (situados entre 1,0 e 2,0), bem como os respectivos desvios-padrão e valores mínimos e máximos.

Ao comparar os resultados encontrados nesta pesquisa com estudo realizado com o cuidador no âmbito hospitalar ${ }^{(8)}$, percebeu-se a presença de 13 itens em comum entre aqueles que obtiveram as médias mais altas (situados entre 6,0 e 5,0) e três itens entre os menores valores médios (situados entre 1,0 e 2,0), conforme pode ser observado na Tabela 3.

No Quadro 1 são apresentados os itens do BEG que estiveram presentes entre os maiores e menores escores em apenas um dos estudos e suas respectivas médias finais $(\mu)$. 
Tabela 1: Distribuição dos maiores escores médios obtidos com o

Questionário de Avaliação do Bem-Estar Global. Maringá, PR, Brasil, 2012.

\begin{tabular}{|c|c|c|c|c|c|}
\hline № & Questões & Média(M) & $\Sigma(D P)$ & Menor & Maior \\
\hline 5. & Minhas crenças me trazem paz de espírito & 5,96 & 0,287 & 4 & 6 \\
\hline 49. & Deus está me ajudando & 5,95 & 0,510 & 1 & 6 \\
\hline 38. & Nós estamos bem em nosso relacionamento & 5,93 & 0,417 & 3 & 6 \\
\hline 8. & Eu sei que sou amado & 5,89 & 0,456 & 4 & 6 \\
\hline 47. & Ela(e) está limpa(o) e seca(o) tanto em casa quanto no hospital & 5,79 & 0,724 & 1 & 6 \\
\hline 18. & Eu sou capaz de me comunicar com quem eu amo & 5,77 & 0,672 & 2 & 6 \\
\hline 21. & Eu tenho pessoas especiais que fazem eu me sentir apoiada(o) & 5,73 & 0,774 & 3 & 6 \\
\hline 2. & Nós não temos privacidade o suficiente* & 5,71 & 0,972 & 1 & 6 \\
\hline 23. & $\begin{array}{l}\text { Eu gosto que o quarto dela(e) esteja tranquilo tanto em casa } \\
\qquad \text { quanto no hospital }\end{array}$ & 5,71 & 0,845 & 1 & 6 \\
\hline 44. & Os amigos se lembram de nós por meio de recados e telefonemas & 5,67 & 0,902 & 1 & 6 \\
\hline 35. & Eu me sinto confiante espiritualmente & 5,63 & 1,098 & 1 & 6 \\
\hline 15. & Eu me sinto culpado* & 5,48 & 1,289 & 1 & 6 \\
\hline 31. & Quando olho para trás vejo que vivemos uma boa vida & 5,47 & 1,289 & 1 & 6 \\
\hline 1. & Meu corpo está relaxado neste momento & 5,41 & 1,302 & 1 & 6 \\
\hline 14. & Eu tenho medo de dormir* & 5,34 & 1,493 & 1 & 6 \\
\hline 19. & Esse quarto me dá medo* & 5,33 & 1,499 & 1 & 6 \\
\hline 33. & $\begin{array}{l}\text { Eu me sinto suficientemente forte para fazer algumas coisas para } \\
\qquad \text { ela(e) }\end{array}$ & 5,23 & 1,318 & 1 & 6 \\
\hline 42. & Eu estou deprimido* & 5,23 & 1,546 & 1 & 6 \\
\hline 3. & Quando eu preciso de ajuda tenho a quem recorrer & 5,20 & 1,374 & 1 & 6 \\
\hline 41. & Eu me sinto capaz de falar para as pessoas aquilo que eu preciso & 5,09 & 1,473 & 1 & 6 \\
\hline 7. & Minha vida não vale a pena nesse momento* & 5,06 & 1,634 & 1 & 6 \\
\hline 27. & Eu posso crescer com essa situação & 5,01 & 1,657 & 1 & 6 \\
\hline
\end{tabular}

* Itens redigidos na forma negativa, que tiveram sua pontuação invertida para análise.

Tabela 2: Distribuição dos menores escores médios obtidos com o Questionário de Avaliação do Bem-Estar Global. Maringá, PR, Brasil, 2012.

\begin{tabular}{rccccc}
\hline No & Questões & Média(M) & $\boldsymbol{\Sigma}(\mathbf{D P})$ & Menor & Maior \\
\hline 4. & Eu me preocupo com minha família* & 1,00 & 0,00 & 1 & 1 \\
45. & O estado emocional dela(e) me deixa triste* & 1,10 & 0,624 & 1 & 6 \\
34. & Eu penso no desconforto dela(e) constantemente* & 1,55 & 1,213 & 1 & 6 \\
48. & Tenho preocupações com a parte financeira* & 1,64 & 1,400 & 1 & 6 \\
46. & Eu penso muito no futuro* & 1,66 & 1,413 & 1 & 6 \\
\hline
\end{tabular}

* Itens redigidos na forma negativa, que tiveram sua pontuação invertida para análise. 
Tabela 3: Itens do Questionário de Avaliação do Bem-Estar Global que apresentaram maiores e menores escores médios na atual pesquisa e no estudo de Rezende et al. ${ }^{(6)}$.

\begin{tabular}{|c|c|c|}
\hline \multirow{2}{*}{ Questões } & \multicolumn{2}{|c|}{ Médias(M) } \\
\hline & Atual & Rezende \\
\hline \multicolumn{3}{|l|}{ Maiores escores } \\
\hline Nós estamos bem em nosso relacionamento & 5,9 & 5,8 \\
\hline Eu sei que sou amado & 5,8 & 5,8 \\
\hline Ela(e) está limpa(o) e seca(o) tanto em casa quanto no hospital & 5,7 & 5,7 \\
\hline Eu sou capaz de me comunicar com quem eu amo & 5,7 & 5,5 \\
\hline Eu tenho pessoas especiais que fazem eu me sentir apoiada(o) & 5,7 & 5,5 \\
\hline Eu gosto que o quarto dela(e) esteja tranquilo tanto em casa quanto no hospital & 5,7 & 5,8 \\
\hline Os amigos se lembram de nós por meio de recados e telefonemas & 5,6 & 5,0 \\
\hline Eu me sinto confiante espiritualmente & 5,6 & 5,5 \\
\hline Eu me sinto culpado(a)* & 5,4 & 5,2 \\
\hline Quando olho para trás vejo que vivemos uma boa vida & 5,4 & 5,6 \\
\hline Eu me sinto suficientemente forte para fazer algumas coisas para ela(e) & 5,2 & 5,6 \\
\hline Quando eu preciso de ajuda tenho a quem recorrer & 5,2 & 5,0 \\
\hline Minha vida não vale a pena nesse momento* & 5,0 & 5,1 \\
\hline \multicolumn{3}{|l|}{ Menores escores } \\
\hline Eu me preocupo com minha família* & 1,0 & 1,1 \\
\hline O estado emocional dela(e) me deixa triste* & 1,1 & 1,6 \\
\hline Eu penso no desconforto dela(e) constantemente* & 1,5 & 1,5 \\
\hline
\end{tabular}

* Itens redigidos na forma negativa, que tiveram sua pontuação invertida para análise.

Quadro 1: Itens do Questionário de Avaliação do Bem-Estar Global que apresentaram maiores e menores escores médios em apenas um dos estudos. Maringá, PR, Brasil, 2012.

\begin{tabular}{|c|c|c|}
\hline & Atual (Média) & Rezende (Média) \\
\hline \multirow{5}{*}{$\begin{array}{l}\text { Maiores } \\
\text { escores }\end{array}$} & $\begin{array}{l}\text { Nós não temos privacidade o } \\
\text { suficiente* }(\mu=5,71)\end{array}$ & As enfermeiras me trazem esperança $(\mu=5,0)$ \\
\hline & $\begin{array}{l}\text { Meu corpo está relaxado neste } \\
\text { momento }(\mu=5,41)\end{array}$ & A temperatura deste quarto é agradável $(\mu=5,0)$ \\
\hline & Eu tenho medo de dormir* $(\mu=5,34)$ & \multirow{3}{*}{ Essa situação me inspira $(\mu=5,2)$} \\
\hline & Esse quarto me dá medo* $(\mu=5,33)$ & \\
\hline & Eu estou deprimido* $(\mu=5,23)$ & \\
\hline \multirow{2}{*}{$\begin{array}{l}\text { Menores } \\
\text { escores }\end{array}$} & \multirow{2}{*}{$\begin{array}{l}\text { Tenho preocupações com a parte } \\
\text { financeira* }(\mu=1,64) \\
\text { Eu penso muito no futuro* }(\mu=1,66)\end{array}$} & Gostaria de ter mais contato com o médico* $(\mu=2,0)$ \\
\hline & & $\begin{array}{l}\text { Eu tenho necessidade de ter mais informações sobre a } \\
\qquad \text { condição dele }(a)^{*}(\mu=2,0)\end{array}$ \\
\hline
\end{tabular}

* Itens redigidos na forma negativa, que tiveram sua pontuação invertida para análise.

\section{DISCUSSÃO}

Apesar de os escores totais de bem-estar global terem apresentados ampla variabilidade, não foram encontrados valores próximos do mínimo da escala, indicando que, de acordo com o instrumento, os níveis de péssimos bem-estar não estavam presentes entre estes cuidadores. Contudo, o BEG não possui estratificação final que possibilite uma classificação dos níveis de bem estar, apenas apontam para os itens que corroboram para a promoção do bem-estar do cuidador, bem como a sua diminuição. Também não existe uma separação clara das dimensões multifatoriais avaliadas, o que dificulta o entendimento e análise dos dados, e pode gerar dúvidas sobre a adequação para estimar escores para fatores específicos. 


\section{Itens que causam maior impacto no bem-estar}

Evidenciou-se que os maiores escores encontrados estão relacionados aos itens que correspondem às crenças espirituais e concepções religiosas e, portanto, esses são os fatores que mais contribuem para a elevação do bem-estar dos cuidadores. Essas questões também apresentaram elevados escores em estudo que trabalhou com os cuidadores durante a hospitalização de seus parentes $^{(8)}$, o que corrobora para a presença da espiritualidade como um evento que auxilia na promoção do bem-estar.

Uma pesquisa de revisão integrativa revelou que são muitos os estudos que apontam para a espiritualidade e religiosidade como sendo uma influência potencial no bem-estar dos cuidadores de pessoas com câncer, por aliviar a pressão, eliminar os temores e possibilitar recursos psicológicos para afrontar e superar tal situação ${ }^{(11)}$. Outro estudo assinalou que muitos são aqueles que, independentemente de sua religião, buscam a cura do câncer na fé, na crença em Deus e na esperança de um milagre ${ }^{(12)}$.

Estes achados podem estar relacionados ao fato de que a espiritualidade e a religião proporcionam aos cuidadores a possibilidade de significação e resposta perante as instabilidades ocasionadas pelo câncer. Desta forma, estes recursos funcionam como um importante aliado no processo de aceitação e enfrentamento da doença, pois conforta, dá esperança e suprem as necessidades emocionais de ter uma expectativa para o futuro.

Outro item que contribuiu para a elevação do bemestar se refere à relação entre cuidador e paciente. Um estudo realizado junto a cuidadores de pacientes com câncer constatou-se a formação de uma relação de cumplicidade e intimidade, que se estabeleceu com o cuidado prestado devido à doença e ao tratamento ${ }^{(13)}$. No entanto, outro trabalho afirmou que a convivência entre o cuidador e o paciente oncológico é cheia de sentimentos opostos, ora de amor, compreensão e solidariedade, ora de raiva, mágoa e frustração, uma vez que o cuidador passa a viver prioritariamente em função do doente ${ }^{(14)}$. Essa exigência reforça a necessidade de uma rede de apoio que auxilie os cuidadores nesta difícil missão.

Tal suporte pode advir de outros integrantes da família, bem como das relações de amizade na comunidade, e dos serviços de assistência social e de saúde. Alguns dos itens encontrados entre os maiores escores do BEG indicavam a presença de pessoas próximas do convívio do cuidador dando-lhe apoio e suporte na prestação dos cuidados.

Estabelecer uma rede de suporte aos cuidadores pode minimizar o sofrimento experenciado e facilitar nos cuidados ao paciente oncológico. No entanto, quando essa rede se dá de maneira precária, o cuidador vivencia uma intensificação do seu desgaste pessoal ${ }^{(11)}$. Por isso, os profissionais que atuam na Estratégia Saúde da Família (ESF), em especial, devem estar atentos para as necessidades enfrentadas pelos cuidadores, capacitandoos para assistir a pessoa com câncer no domicílio e orientando-os de acordo com sua realidade.

Sentir-se seguro na prestação de cuidados é um evento que eleva o bem-estar. Em geral, na grande maioria dos casos, os cuidadores são pessoas que não possuem formação específica que lhes permita garantir a qualidade dos cuidados, nem estão emocionalmente preparados para assumir os encargos desta função. Destarte, a presença do enfermeiro no domicílio torna-se indispensável, ouvindo, olhando e interpretando queixas e sinais que porventura surjam e tomando decisões pertinentes, pois sem este valioso instrumento, que é a visita domiciliar, o enfermeiro não poderá realizar uma assistência integral ao paciente oncológico e sua família.

$\mathrm{O}$ ato de cuidar da higiene corporal do familiar com câncer sugere que manter o doente asseado constitui-se em um fator importante para o bem-estar do cuidador, assim como a constatação de tranquilidade no quarto do paciente. Esses dados sugerem a importância dos efeitos do ambiente para o bem-estar dos cuidadores e reforça a relevância de investimentos em espaço e arquitetura ${ }^{(15)}$. 
Muitas vezes são medidas simples, concretas e rápidas que tornam um ambiente mais tranquilo e acolhedor, o respeito ao silêncio, uma iluminação indireta, uma adequada escolha de cores, boa ventilação, limpeza, dentre outras ${ }^{(8)}$.

A questão "Eu me sinto culpado"* era uma das quais possuía redação negativa, e teve o valor de média final invertido. Assim, a sensação de não se sentir culpado está associada ao bem-estar do cuidador. Corroborando com este pensar, um estudo afirmou que o fato de sentir-se responsável pelo adoecimento do seu familiar, influencia negativamente os cuidadores podendo atuar como um elemento estressor e desencadear maiores índices de estresse $^{(15)}$.

Outro item que foi apontado como favorável ao bemestar se refere às relembranças positivas do passado. Esse dado indica que ao contemplar o passado e lembrar-se do familiar enfermo com saúde, da antiga rotina familiar e dos momentos felizes que passaram, faz com que os cuidadores tenham a sensação de bem-estar. Logo, a lembrança do passado é um assunto que traz satisfação e pode ser introduzido pela equipe de saúde durante a visita domiciliar, uma vez que o diálogo com o cuidador não deve ser pautado apenas na doença do familiar com câncer, mas em assuntos que lhe tragam alegria.

A elevação do bem-estar também esteve relacionada à percepção de poder ajudar o familiar que está com a saúde debilitada. Daí a importância de valorizar a participação dos cuidadores no processo de cuidar, incluindo-os durante as tarefas de cuidado no domicílio.

\section{Itens que causam menor impacto no bem-estar}

A preocupação com a família foi o fator que mais teve impacto negativo no bem-estar global dos cuidadores. Tal resultado levanta a hipótese de que os cuidadores se inquietam com outras questões relacionadas às suas famílias, o que gera sobrecarga emocional ${ }^{(16)}$.

Presenciar o desconforto físico e emocional, e o pensamento constante no indivíduo com câncer também foram aspectos que contribuíram para a diminuição do bem-estar. Ao vivenciar o agonia do ser com câncer, o cuidador passa a compartilhar da dor física do outro ${ }^{(17)}$. Essa experiência traz dor, principalmente quando se trata de alguém muito próximo. Tal situação faz com que os familiares se enterneçam diante do sofrimento de alguém tão querido e sofram constantemente.

\section{Itens presentes entre os maiores e menores escores em apenas um dos estudos}

Cinco itens estiveram presentes apenas no estudo atual entre os maiores escores e sugerem que o cuidado realizado no lar possibilita maior calmaria e privacidade para o cuidador familiar, diminuindo os sintomas de estresse (ansiedade, medo, depressão e dificuldade para dormir). Essa percepção corrobora com uma pesquisa realizada junto à cuidadores de paciente com câncer, o qual apontou que mesmo diante de todas as dificuldades enfrentadas no domicílio, a maioria deles preferiu cuidar do familiar doente em casa do que no hospital ${ }^{(18)}$.

Três itens estiveram presentes apenas entre os maiores escores no estudo de Rezende et al. ${ }^{(8)}$. Infere-se que o fato de os cuidadores reconhecerem as enfermeiras como fonte de esperança, pode estar relacionado à presença constante deste profissional dentro do hospital, ou ainda, pela ausência do enfermeiro da Atenção Básica no cuidado ao paciente oncológico e seus familiares.

Nota-se a existência de uma grande lacuna quando se fala em acompanhamento dos serviços de saúde à pessoa com câncer no domicílio. Há, portanto, a necessidade de ampliação dos serviços de atenção domiciliária nas suas diversas modalidades, uma vez que esse apoio tem sido insuficiente e não oferece suporte e ajuda efetiva aos cuidadores. Assim, surge à necessidade de implementação de políticas públicas que ajudem a manter o indivíduo com câncer no domicílio.

Os itens que estiveram presentes apenas na atual pesquisa entre os menores escores, indicam incerteza em relação à parte financeira. Estudo aponta que o câncer é responsável pelo declive financeiro de muitas famílias, já que os custos com o tratamento são altos, o paciente 
deixam de trabalhar e o cuidador também interrompe ou diminui sua jornada, a fim de assistir o familiar doente ${ }^{(3,18)}$.

Por fim, dois itens estiveram presentes entre os menores escores apenas no estudo de Rezende et al. ${ }^{(8)}$. Tal resultado pode ser explicado pelo fato de que, apesar da presença constante dos profissionais de saúde dentro do hospital, nem sempre esses estão dispostos a esclarecer as dúvidas suscitados pelos cuidadores. O que se vê, muitas vezes, é que essas informações são transmitidas de forma insuficientes e não são compreendidas pelos acompanhantes, resultando em diminuição do bem-estar dos cuidadores.

\section{CONCLUSÃO}

O presente estudo é uma oportunidade para refletir sobre a importância de se incluir o cuidador como participante do processo de cuidar. Os profissionais de saúde devem compreender e estimular as práticas religiosas e espirituais da família, uma vez que a avaliação e a intervenção espiritual devem ser parte do cuidado holístico; devem buscar alternativas para a construção de uma rede de apoio ao cuidador, de modo que esses indivíduos possam conciliar as atividades relativas ao cuidado e a outras atividades, e ainda; auxiliar os cuidadores de forma efetiva durante o planejamento das tarefas de cuidado no domicílio, com sugestões que facilitem o seu cotidiano, bem como na organização do ambiente de cuidado.

Apesar de ser realizada em local diferente e utilizar amostra sociodemográfica diferente, o estudo de Rezende et al. apontou semelhanças com os resultados obtidos neste estudo, o que indica que o cenário interfere pouco em relação ao bem estar do cuidador, ou seja, independente do local seu bem estar tem o mesmo comprometimento. Contudo, alguns itens presentes no estudo atual, podem sugerir que cuidar no domicílio tem impacto maior no bem-estar dos cuidadores, em comparação a cuidar no hospital.

O questionário utilizado serve como instrumento de apoio no planeamento das intervenções de enfermagem. Mas, faz se necessário à criação de outros instrumentos que forneçam uma avaliação rápida e eficaz das necessidades dos cuidadores de pacientes oncológicos no domicilio.

Como limitações deste estudo, consideram-se as dificuldades na realização da coleta dos dados, pela impossibilidade de contato telefônico - número incorreto ou desativado; poucas publicações relacionadas ao bemestar de cuidadores, e a possibilidade do viés de seleção, pois apesar dos cuidados para se evitar que tivéssemos uma amostra tendenciosa, trata-se de um grupo nãorepresentativo, por estarem inseridos em uma organização destinada a atender pacientes oncológicos com poucas condições financeiras.

Estudos futuros relacionados com o Bem-Estar Global de cuidadores de adultos com neoplasias malignas no domicílio ainda são necessários, visando maior compreensão da problemática em questão e à melhoria do atendimento a esses indivíduos. Sugerem-se, então, estudos com aplicação de análises estatísticas correlacionais e multivariadas, com objetivo de analisar a relação entre o Bem-Estar Global e as características pessoais dos cuidadores e dos indivíduos com neoplasias malignas.

3. Maronesi LC, Silva NR, Cantu SO, Santos AR. Indicadores de estresse e sobrecarga em cuidadores formais e informais de pacientes oncológicos. Estud. pesqui. psicol. 2014;14(3):877-892.

4. Anjos ACY, Zago MMF. Ressignificação da vida do cuidador do paciente idoso com câncer. Rev Bras Enferm. 2014;67(5):752-8. 5. Santo EARE, Gaíva MAM, Espinosa MM, Barbosa DA, Belasco AGS. Taking care of children with cancer: evaluation of the caregivers' 
burden and quality of life. Rev Latino-Am Enfermagem.

2011;19(3):515-22.

6. Rezende VL, Derchain SM, Botega NJ, Vial DL. Revisão crítica dos instrumentos utilizados para avaliar aspectos emocionais, físicos e sociais do cuidador de pacientes com câncer na fase terminal da doença. Rev Bras Cancerol. 2005;51(1):79-87.

7. Kolcaba K. Comfort theory and practice: a vision for holistic health care and research. New York: Springer Publishing Company, 2003.

8. Rezende VL, Derchain S, Botega NJ, Sarian LO, Vial DL, Morais SS, Perdicaris AAM. Avaliação psicológica dos cuidadores de mulheres com câncer pelo General Comfort Questionnaire. Paidéia. 2010;20(46):22937.

9. Lamino DA, Turrini RNT, kolcaba k. Conforto De Cuidadores De Pacientes Com Câncer. Rev. esc. enferm. USP. 2014;48(2):278-284. 10. Moreira LM, Ferreira RA, costa Junior AL. Discussão de protocolo para cuidadores de pacientes com câncer em cuidados paliativos. Paidéia (Ribeirão Preto) [online]. 2012;22(53):383-392.

11. Sanchez KOL, Ferreira NMLA, Dupas G, Costa DB. Apoio social à família do paciente com câncer: identificando caminhos e direções. Rev Bras Enferm. 2010;63(2):290-99.

12. Guerrero GP, Zago MMF, Sawada NO, Pinto, MH. Relação entre espiritualidade e câncer: perspectiva do paciente. Rev Bras Enferm. 2011;64(1):53-9.

13. Sales CA, Piolli KC, Reticena KO, Wakiuchi J, Marcon SS. Presença familiar no olhar existencial da pessoa com câncer: compreendendo o fenômeno à luz heideggeriana. Rev. Eletr. Enf. [Internet].

2015;17(1):30-6.

14. Fernandes JJBR. Cuidar no domicílio e a sobrecarga do cuidador familiar [Dissertação de mestrado]. Lisboa (Portugal): Faculdade de Medicina da Universidade de Lisboa; 2009. 155 p.

15. Faria AMDB, Cardoso CL. Aspectos psicossociais de acompanhantes cuidadores de crianças com câncer: stress e enfrentamento. Estud Psicol. 2010;27(1):13-20.

16. Souza MGG, Santo FHE. O olhar que olha o outro... um estudo com familiares de pessoas em quimioterapia antineoplásica. Rev Bras Cancerol. 2008;53(1):31-41.

17. Sena ELS, Carvalho PAL, Reis HFT, Rocha MB. Percepção de familiares sobre o cuidado à pessoa com câncer em estágio avançado. Texto \& Contexto Enferm. 2011;20(4):774-81.

18. Carvalho CSU. A necessária atenção à família do paciente oncológico. Rev Bras Cancerol. 2008;54(1):87-96.

Recebido: 04/03/2014.

Aceito: 04/03/2015.

Publicado: 30/06/2015. 